\title{
Multiple common variants for celiac disease influencing immune gene expression
}

Patrick CA Dubois ${ }^{1,37}$, Gosia Trynka ${ }^{2,37}$, Lude Franke ${ }^{1,2}$, Karen A Hunt ${ }^{1}$, Jihane Romanos ${ }^{2}$, Alessandra Curtotti ${ }^{3}$, Alexandra Zhernakova ${ }^{4}$, Graham AR Heap ${ }^{1}$, Róza Ádány ${ }^{5}$, Arpo Aromaa $^{6}$, Maria Teresa Bardella ${ }^{7,8}$, Leonard H van den Berg ${ }^{9}$, Nicholas A Bockett ${ }^{1}$, Emilio G. de la Concha ${ }^{10}$, Bárbara Dema ${ }^{10}$, Rudolf SN Fehrmann², Miguel Fernández-Arquero ${ }^{10}$, Szilvia Fiatal ${ }^{5,11}$, Elvira Grandone ${ }^{12}$, Peter M Green ${ }^{13}$, Harry JM Groen ${ }^{14}$, Rhian Gwilliam ${ }^{15}$, Roderick HJ Houwen ${ }^{16}$, Sarah E Hunt ${ }^{15}$, Katri Kaukinen ${ }^{17}$, Dermot Kelleher ${ }^{18}$, IIma Korponay-Szabo ${ }^{19,20}$, Kalle Kurppa ${ }^{17}$, Padraic MacMathuna ${ }^{21}$, Markku Mäki ${ }^{17}$, Maria Cristina Mazzilli ${ }^{22}$, Owen T McCann ${ }^{15}$, M Luisa Mearin ${ }^{23}$, Charles A Mein ${ }^{3}$, Muddassar M Mirza $^{13}$, Vanisha Mistry ${ }^{1}$, Barbara Mora ${ }^{22}$, Katherine I Morley ${ }^{15}$, Chris $\mathrm{J} \mathrm{Mulder}^{24}$, Joseph A Murray ${ }^{25}$, Concepción Núñez ${ }^{10}$, Elvira Oosterom ${ }^{2}$, Roel A Ophoff ${ }^{26,27}$, Isabel Polanco ${ }^{28}$, Leena Peltonen ${ }^{15,29}$, Mathieu Platteel ${ }^{2}$, Anna Rybak ${ }^{30}$, Veikko Salomaa ${ }^{6}$, Joachim J Schweizer ${ }^{23}$, Maria Pia Sperandeo ${ }^{31}$, Greetje $\mathbf{J ~ T a c k}^{24}$, Graham Turner ${ }^{18}$, Jan H Veldink ${ }^{9}$, Wieke HM Verbeek ${ }^{24}$, Rinse K Weersma ${ }^{2,32}$, Victorien M Wolters ${ }^{16}$, Elena Urcelay ${ }^{10}$, Bozena Cukrowska ${ }^{33}$, Luigi Greco ${ }^{31}$, Susan L. Neuhausen ${ }^{34}$, Ross McManus ${ }^{18}$, Donatella Barisani $^{35}$, Panos Deloukas ${ }^{15}$, Jeffrey C Barrett ${ }^{15}$, Paivi Saavalainen ${ }^{36}$, Cisca Wijmenga ${ }^{2}$, and David A van Heel ${ }^{1}$

${ }^{1}$ Blizard Institute of Cell and Molecular Science, Barts and The London School of Medicine and Dentistry, Queen Mary University of London, London E1 2AT, UK ${ }^{2}$ Genetics Department, University Medical Center and Groningen University, 9700 RB Groningen, The Netherlands ${ }^{3}$ The Genome Centre, Barts and The London School of Medicine and Dentistry, Queen Mary University of London, London EC1M 6BQ, UK ${ }^{4}$ Division of Biomedical Genetics, University Medical Center Utrecht, Utrecht, The Netherlands ${ }^{5}$ Department of Preventive Medicine, University of Debrecen, Debrecen, Hungary ${ }^{6}$ National Institute for Health and Welfare, Helsinki 00271, Finland ${ }^{7}$ Fondazione IRCCS Ospedale Maggiore Policlinico, Mangiagalli e Regina Elena, Milan, Italy ${ }^{8}$ Department of Medical Sciences, University of Milan, Italy ${ }^{9}$ Department of Neurology, Rudolf Magnus Institute of Neuroscience, University Medical Centre Utrecht, Heidelbergaan 100, Utrecht, The Netherlands ${ }^{10}$ Clinical Immunology Department, Hospital Clínico San Carlos, Madrid, Spain ${ }^{11}$ Public Health Research Group of Hungarian Academy of Sciences, Medical \& Health Science Center, University of Debrecen, Debrecen, Hungary ${ }^{12}$ Unita' di Aterosclerosi e Trombosi, I.R.C.C.S Casa Sollievo della Sofferenza, S. Giovanni Rotondo, Foggia, Italy ${ }^{13} \mathrm{NIHR}$ GSTFT/KCL Comprehensive Biomedical Research Centre King's College London School of Medicine 7th Floor Tower Wing Guy's Hospital London SE19RT United Kingdom ${ }^{14}$ Dept. of

Correspondence to DAvH (d.vanheel@qmul.ac.uk) or regarding expression analyses to LF (lude@ ludesign.nl)..

${ }^{37}$ These authors contributed equally

AUTHOR CONTRIBUTION STATEMENT

$\mathrm{DAvH}$ and $\mathrm{CW}$ designed, co-ordinated and led the study. Experiments were performed in the labs of CW, DAvH, CAM, PD and PMG. Major contributions were: 1) DNA sample preparation - PCAD, GT, KAH, JR, AZ, PS 2) genotyping - PCAD, GT, KAH, AC, JR, RG; 3) expression data generation - HJMG, LHvdB, RAO, RKW, LF; 4) case/control association analyses - PCAD, GT, LF, JCB, DAvH; 5) expression analyses - LF, GAH, RSNF; 6) manuscript preparation - PCAD, GT, LF, RSNF, GAH, JCB, CW, DAvH. Other authors contributed variously to sample collection and all other aspects of the study. All authors reviewed the final manuscript.

The authors declare no competing financial interests.

DATABASE ACCESSION NUMBERS

Expression data is available in GEO (http://www.ncbi.nlm.nih.gov/geo/) as GSE11501 and GSE20142. 
Pulmonology, University Medical Center and Groningen University, 9700 RB Groningen, The Netherlands ${ }^{15}$ Wellcome Trust Sanger Institute, Hinxton, Cambridge CB10 1SA, UK

${ }^{16}$ Department of Paediatric Gastroenterology, University Medical Centre Utrecht, Utrecht, The Netherlands ${ }^{17}$ Paediatric Research Centre, University of Tampere Medical School and Tampere University Hospital, Tampere, Finland ${ }^{18}$ Department of Clinical Medicine, Institute of Molecular Medicine, Trinity College Dublin, Dublin, Ireland ${ }^{19} \mathrm{Heim}$ Pal Childrens Hospital, Budapest, Hungary ${ }^{20}$ Department of Pediatrics, Medical and Health Science Center, University of Debrecen, Hungary ${ }^{21}$ Gastrointestinal Unit, Mater Misericordiae University Hospital, Eccles St., Dublin 7, Ireland ${ }^{22}$ Department of Experimental Medicine, Sapienza University of Rome, Rome, Italy ${ }^{23}$ Department of Paediatrics, Leiden University Medical Centre, Leiden, The Netherlands ${ }^{24}$ Department of Gastroenterology, VU Medical Center, 1007 MB Amsterdam, The Netherlands ${ }^{25}$ Division of Gastroenterology and Hepatology, Department of Medicine, Mayo Clinic College of Medicine, Rochester, MN 55905, USA ${ }^{26}$ Department of Medical Genetics and Rudolf Magnus Institute, University Medical Center Utrecht, Utrecht, The Netherlands ${ }^{27}$ Center for Neurobehavioral Genetics, University of California, Los Angeles, CA, USA ${ }^{28}$ Pediatric Gastroenterology Department, Hospital La Paz, Madrid, Spain ${ }^{29}$ Institute for Molecular Medicine Finland, FIMM, Helsinki 00290, Finland ${ }^{30}$ Department of Gastroenterology, Hepatology and Immunology, Children's Memorial Health Institute, Warsaw, Poland ${ }^{31}$ European Laboratory for Food Induced Disease, University of Naples Federico II, Naples, Italy ${ }^{32}$ Department of Gastroenterology and Hepatology, University Medical Centre Groningen, University of Groningen, Groningen, The Netherlands ${ }^{33}$ Department of Pathology, Children's Memorial Health Institute, Warsaw, Poland ${ }^{34}$ Department of Population Sciences, Beckman Research Institute of the City of Hope, Duarte, CA, USA ${ }^{35}$ Department of Experimental Medicine, Faculty of Medicine University of Milano-Bicocca, Monza, Italy ${ }^{36}$ Department of Medical Genetics \& Research Program for Molecular Medicine, Biomedicum Helsinki, University of Helsinki, Helsinki, Finland

\section{Abstract}

We performed a second-generation genome wide association study of 4,533 celiac disease cases and 10,750 controls. We genotyped 113 selected SNPs with $P_{\mathrm{GWAS}}<10^{-4}$, and 18 SNPs from 14 known loci, in a further 4,918 cases and 5,684 controls. Variants from 13 new regions reached genome wide significance $\left(P_{\text {combined }}<5 \times 10^{-8}\right)$, most contain immune function genes $(B A C H 2$, CCR4, CD80, CIITA/SOCS1/CLEC16A, ICOSLG, ZMIZ1) with ETS1, RUNX3, THEMIS and TNFRSF14 playing key roles in thymic T cell selection. A further 13 regions had suggestive association evidence. In an expression quantitative trait meta-analysis of 1,469 whole blood samples, 20 of $38(52.6 \%)$ tested loci had celiac risk variants correlated $(P<0.0028$, FDR 5\%) with cis gene expression.

Celiac disease is a common heritable chronic inflammatory condition of the small intestine induced by dietary wheat, rye and barley, as well as other unidentified environmental factors, in susceptible individuals. Specific $H L A-D Q A 1$ and $H L A-D Q B 1$ risk alleles are necessary, but not sufficient, for disease development1,2. The well defined role of HLA-DQ heterodimers, encoded by these alleles, is to present cereal peptides to $\mathrm{CD}^{+} \mathrm{T}$ cells, activating an inflammatory immune response in the intestine. A single genome wide association study (GWAS) has been performed in celiac disease, which identified the IL2/ IL21 risk locus1. Subsequent studies probing the GWAS information in greater depth have identified a further 12 risk regions. Most of these regions contain a candidate gene functional in the immune system, although only in the case of $H L A-D Q A 1$ and $H L A-D Q B 1$ have the causal variants been established3-5. Many of the known celiac loci overlap with other immune-related diseases6. In order to identify additional risk variants, particularly of smaller effect size, we performed a second-generation GWAS using over six times as many 
samples as the previous GWAS and a denser genome-wide SNP set. We followed up promising findings in a large collection of independent samples.

The GWAS included five European celiac disease case and control sample collections including the previously reported celiac disease dataset1. We performed stringent data quality control (Online Methods), including calling genotypes using a custom algorithm on both large sample sets, and where possible cases and controls together (Online Methods). We tested 292,387 non-HLA SNPs from the Illumina Hap300 marker set for association in 4,533 celiac disease cases and 10,750 controls of European descent (Table 1). A further 231,362 additional non- $H L A$ markers from the Illumina Hap550 marker set were tested for association in a subset of 3,796 celiac disease cases and 8,154 controls. All markers were from autosomes or the $\mathrm{X}$ chromosome. Genotype call rates were $>99.9 \%$ in both datasets. The overdispersion factor of association test statistics, $\lambda_{\mathrm{GC}}=1.12$, was similar to that observed in other GWAS of this sample size7,8. Findings were not substantially altered by imputation of missing genotypes for 737 celiac disease cases genotyped on the Hap300 BeadChip and corresponding controls (Table 1, collection 1). Here we present results for directly genotyped samples, as around half the additional Hap550 markers cannot be accurately imputed from Hap300 data9 (including the new ETS1 locus finding in this study). Results for the top 1000 markers are available in Supplementary Data 1, but because of concerns regarding identity detection of individuals 10, results for all markers are available only on request to the corresponding author.

For follow-up, we first inspected genotype clouds for the 417 non- HLA SNPs meeting $P_{\mathrm{GWAS}}<10^{-4}$, being aware that top GWAS association signals may be enriched for genotyping artefact, and excluded 22 SNPs from further analysis using a low threshold for possible bias. We selected SNPs from 113 loci for replication. Markers that passed design and genotyping quality control included: a) 18 SNPs from all 14 previously identified celiac disease risk loci (including a tag SNP for the major celiac disease associated HLA-DQ2.5cis haplotype1); b) 13 SNPs from all 7 novel regions with $P_{\mathrm{GWAS}}<5 \times 10^{-7}$; c) 86 SNPs from 59 of 68 novel regions with $5 \times 10^{-7}>P_{\mathrm{GWAS}}>5 \times 10^{-5}$ in stage 1 ; d) 14 SNPs from 14 of 30 novel regions with $5 \times 10^{-5}>P_{\mathrm{GWAS}}>10^{-4}$ in stage 1 (for this last category, we mostly chose regions with immune system genes). Two SNPs were selected per region for: regions with stronger association; regions with possible multiple independent associations; and/or containing genes of obvious biological interest. We successfully genotyped 131 SNPs in 7 independent follow-up cohorts comprising 4,918 celiac disease cases and 5,684 controls of European descent. Genotype call rates were $>99.9 \%$ in each collection. Primary association analyses of the combined GWAS and follow-up data were performed with a two-sided $2 \times 2 \times 12$ Cochran-Mantel-Haenszel test.

\section{RESULTS}

\section{Celiac disease risk variants}

The $H L A$ locus and all 13 other previously reported celiac disease risk loci showed evidence for association at a genome wide significance threshold $\left(P_{\text {combined }}<5 \times 10^{-8}\right)$ (Table 2$)$. We note that some loci were previously reported using less stringent criteria (e.g. the $P<5 \times 10^{-7}$ recommended by the 2007 WTCCC study 11), but that in the current, much larger sample set, all known loci meet recently proposed $P<5 \times 10^{-8}$ thresholds 12,13 .

We identified 13 novel risk regions with genome-wide significant evidence $\left(P_{\text {combined }}\right.$ $<5 \times 10^{-8}$ ) of association, including regions containing the $B A C H 2, C C R 4, C D 80, C I I T A$ / SOCS1/CLEC16A, ETS1, ICOSLG, RUNX3, THEMIS, TNFRSF14, and ZMIZ1 genes which are of obvious immunological function (Table 2). A further 13 regions met 'suggestive' criteria for association (either $10^{-6}>P_{\text {combined }}>5 \times 10^{-8}$ and/or $P_{\text {GWAS }}<10^{-4}$ 
and $\left.P_{\text {followup }}<0.01\right)$. These regions also contain multiple genes of obvious immunological function, including CD247, FASLGITNFSF18/TNFSF4, IRF4, TLR7/TLR8, TNFRSF9 and $Y D J C$. Six of the 39 non-HLA regions show evidence for the presence of multiple independently associated variants in a conditional logistic regression analysis (Supplementary Table 2).

We tested the 40 SNPs with the strongest association (Table 2) from each of the known genome-wide significant, new genome-wide significant, and new suggestive loci for evidence of heterogeneity across the 12 collections studied. Only the $H L A$ region was significant (Breslow-Day test $P<0.05 / 40$ tests, rs $2187668 P=4.8 \times 10^{-8}$ ) which is consistent with the well described North-South gradient in HLA allele frequency in European populations, and more specifically for $H L A-D Q$ in celiac disease 14 .

We observed no evidence for interaction between each of the 26 genome-wide significant non- $H L A$ loci, which is consistent with what has been reported for complex diseases so far. However, we did observe weak evidence for lower effect sizes at non- $H L A$ loci in high risk $H L A-D Q 2.5$ cis homozygotes, similar to what has been previously observed in type 1 diabetes7.

To obtain more insight into the functional relatedness of the celiac loci, we applied GRAIL, a statistical tool that utilizes text mining of PubMed abstracts to annotate candidate genes from loci associated with common disease risk 15,16 . In order to assess the sensitivity of this tool (using known loci as a positive control), we first performed a 'leave-one-out' analysis of the 27 genome-wide significant celiac disease loci (including $H L A-D Q$ ). GRAIL scores of $P_{\text {text }}<0.01$ were obtained for 12 of the 27 loci (44\% sensitivity, Table 2). Factors that limit the sensitivity of GRAIL include biological pathways being both known (a 2006 dataset is used to avoid GWAS era studies), and published in the literature. We then applied GRAIL analysis, using the 27 known regions as a seed, to all 49 regions (49 SNPs) with $10^{-3}>P_{\text {combined }}>5 \times 10^{-8}$ and obtained GRAIL $P_{\text {text }}<0.01$ for 9 regions $(18.4 \%)$. As a control, only $5.5 \%$ (279 of 5033) of randomly selected Hap550 SNPs reached this threshold. In addition to the five 'suggestive' loci shown in Table 2, GRAIL annotated four further interesting gene regions of lower significance in the combined association results: rs944141/ $P D C D 1 L G 2\left(P_{\text {combined }}=4.4 \times 10^{-6}\right)$, rs $976881 / T N F R S F 8\left(\mathrm{P}_{\text {combined }}=2.1 \times 10^{-4}\right), \mathrm{rs} 4682103 /$ $C D 200 / B T L A\left(P_{\text {combined }}=6.8 \times 10^{-6}\right)$ and $\mathrm{rs} 4919611 /$ NFKB2 $\left(P_{\text {combined }}=6.1 \times 10^{-5}\right)$. There appeared to be further enrichment for genes of immunological interest which are not GRAIL annotated in the $10^{-3}>P_{\text {combined }}>5 \times 10^{-8}$ significance window, including rs3828599/TNIP1 $\left(P_{\text {combined }}=1.55 \times 10^{-4}\right)$, rs8027604/PTPN9 $\left(P_{\text {combined }}=1.4 \times 10^{-6}\right)$, rs $944141 / C D 274$ $\left(P_{\text {combined }}=4.4 \times 10^{-6}\right)$. Some of these findings, for which neither genome-wide significant nor suggestive association is achieved, are likely to comprise part of a longer tail of disease predisposing common variants, of weaker effect sizes. Definitive assessment of these biologically plausible regions would require genotyping and association studies using much larger sample collections than the present study.

We previously showed considerable overlap between celiac disease and type 1 diabetes risk loci17, as well as celiac disease and rheumatoid arthritis18, and more generally, there is now substantial evidence for shared risk loci between the common chronic immune mediated diseases6. To update these observations, we searched 'A Catalog of Published Genome Wide Association Studies' (18 Nov 2009)19 and the HuGE database20. We found some evidence (requiring a published association report of $P<1 \times 10^{-5}$ ) of shared loci with at least one other inflammatory or immune mediated disease for 18 of the current 27 genome-wide significant celiac risk regions. We defined shared regions as the broad LD block, however different SNPs are often reported in different diseases, and at only three of the 18 shared regions are associations across all diseases with the same SNP or a proxy SNP in $\mathrm{r}^{2}>0.8$ in 
HapMap CEU. Currently 9 regions appear celiac disease specific and may reflect distinctive disease biology including the regions containing rs296547 and rs9792269, and the regions around CCR4, CD80, ITGA4, LPP, PLEK, RUNX3 and THEMIS. In fact, locus sharing between diseases is probably greater, due to both stochastic variation in results from sample size limitations, and regions with a genuinely stronger effect size in one disease and weaker effect size in another.

Genetic variation in ETS1 has recently been reported to be associated with systemic lupus erythematosus (SLE) in the Chinese population, although is not associated with SLE in European populations 21 . The most strongly associated celiac (European population) SNP rs11221332 and the most strongly associated SLE (Chinese population) SNP rs6590330 map $70 \mathrm{~kb}$ apart. Inspection of the HapMap phase II data shows broadly similar linkage disequilibrium patterns between Chinese (CHB) and European (CEU) populations in this region, with the two associated SNPs in separate non-adjacent linkage disequilibrium blocks. Thus distinct common variants within the same gene are predisposing to different autoimmune diseases across different ethnic groups.

\section{Function of celiac risk variants}

Celiac risk variants in the $H L A$ alter protein structure and function4. However we identified only four non-synonymous SNPs with evidence for celiac disease association $\left(P_{\mathrm{GWAS}}<10^{-4}\right)$ from the other 26 genome-wide significant associated regions (rs3748816/ MMEL1, rs3816281/PLEK, rs196432/RUNX3, rs3184504/SH2B3). Although comprehensive regional resequencing is required to test the possibility that coding variants contribute to the observed association signals, more subtle effects of genetic variation on gene expression are the more likely major functional mechanism for complex disease genes. With this in mind, we performed a meta-analysis of new and published genome-wide expression quantitative trait loci (eQTL) datasets comprising 1,469 human whole blood (PAXgene) samples reflecting primary leucocyte gene expression. We applied a new method, Transcriptional Components, to remove a substantial proportion of inter-individual non-genetic expression variation and performed eQTL meta-analysis on the residual expression variation (Online Methods).

We assessed 38 of the 39 genome-wide significant and suggestive celiac disease associated non- HLA loci (Table 2) for cis expression - genotype correlations. We tested the SNP with the strongest association from each region. However for five regions the most associated SNP was not genotyped in the eQTL samples (Hap300 data), instead for four of these we tested a proxy SNP $\left(r^{2}>0.5\right.$ in HapMap CEU). In addition, for six loci showing evidence of multiple independent associations in conditional regression analyses, we tested a second SNP showing independent celiac disease association for eQTL analysis. In total we assessed 44 independent non- $H L A$ SNP associations in peripheral whole blood samples genotyped on the Illumina Hap300 BeadChip and either Illumina Ref8 or HT12 expression arrays, correlating each SNP with data from gene-probes mapping within a $1 \mathrm{Mb}$ window.

We identified significant (Spearman $P<0.0028$, corresponding to 5\% false discovery rate) eQTLs at 20 of $38(52.6 \%)$ non- $H L A$ celiac loci tested (Table 3, Supplementary Figures $2 \&$ 3). Some loci had evidence of eQTLs with multiple probes, genes or SNPs (Table 3). We assessed whether the number of SNPs with cis-eQTL effects out of the 44 SNPs that we tested, was significantly higher than expected. We observed that eQTL SNPs on average have a substantially higher MAF than non-eQTL SNPs in the 294,767 SNPs tested. In order to correct for this we selected 44 random SNPs that had an equal MAF distribution, and determined for how many of these MAF-matched SNPs eQTLs were observed. We observed a significantly higher number of eQTL SNPs $\left(P=9.3 \times 10^{-5}, 10^{6}\right.$ permutations $)$ amongst the celiac associated SNPs than expected by chance (22 observed eQTL SNPs, vs. 7.8 expected 
eQTL SNPs). Therefore the celiac disease associated regions are greatly enriched for eQTLs. These data suggest some risk variants may influence celiac disease susceptibility through a mechanism of altered gene expression. Candidate genes with a significant eQTL, where the peak eQTL signal and peak case/control association signal are similar (Supplementary Figure 3), include MMEL1, NSF, PARK7, PLEK, TAGAP, RRP1, $U B E 2 L 3$ and ZMIZ1.

We also assessed co-expression of genes mapping within 500kb of SNPs showing strongest case/control association from the 40 genome-wide significant and suggestive celiac disease loci in an analysis of the 33,109 human Affymetrix Gene Expression Omnibus dataset. This analysis loses power to detect tissue specific correlations by use of numerous tissue types, but greatly gains power by the large sample size. We detected several distinct co-expression clusters (Pearson correlation coefficient between genes $>0.5$ ), including four clusters of immune-related genes which contain at least one gene from 37 of the 40 genome-wide significant and suggestive loci (Fig. 1). These data further demonstrate that genes from celiac disease risk loci map to multiple distinct immunological pathways involved in disease pathogenesis.

\section{DISCUSSION}

We previously reported that most celiac genetic risk variants mapped near genes that are functional in the immune system 22 , and this remains true for the 13 new genome-wide significant, and 13 new suggestive, risk variants from the current study. We can now refine these observations and highlight specific immunological pathways relevant to celiac disease pathogenesis:

\section{1) T cell development in the thymus}

The rs 802734 LD block contains the recently identified gene THEMIS 'THymus-Expressed Molecule Involved in Selection'. THEMIS plays a key regulatory role in both positive and negative T-cell selection during late thymocyte development23. Furthermore, the rs 10903122 LD block contains $R U N X 3$, a master regulator of CD8 ${ }^{+}$T lymphocyte development in the thymus24,25. TNFRSF14 (LIGHTR, rs3748816 LD block) has widespread peripheral leucocyte functions as well as a critical role in promoting thymocyte apoptosis26. The ETS1 transcription factor (rs11221332 LD block) is also active in peripheral leucocytes, however it is also a key player in thymic CD8 ${ }^{+}$lineage differentiation, acting in part by promoting $R U N X 3$ expression 27.

The importance of the thymus in autoimmune disease pathogenesis has been previously emphasised by the established role of thymectomy in the treatment of myasthenia gravis. In type 1 diabetes, it was shown that disease associated genetic variation in the insulin gene INS causes altered thymic insulin expression and subsequent $\mathrm{T}$ cell tolerance for insulin as a self-protein 28 . However, the importance of thymic $\mathrm{T}$ cell regulation has not been previously recognised in the aetiology of celiac disease. It is conceivable that the associated variants may alter biological processes prior to thymic MHC-ligand interactions. Alternatively it is now clear that exogenous antigen presentation and selection occurs in the thymus via migratory dendritic cells - this has been demonstrated for skin, and has been hypothesised for food antigens29,30. These findings suggest research into immuno-/pharmaco-logical modifiers of $\mathrm{T}$ cell tolerance more generally in autoimmune diseases.

\section{2) Innate immune detection of viral RNA}

Although the association signal at rs5979785 $\left(P_{\text {combined }}=6.36 \times 10^{-8}\right)$ in the TLR7/TLR 8 region is just outside our genome wide significance threshold, we observe a strong effect of 
rs5979785 on TLR8 expression in whole blood. Both TLRs recognise viral RNA. Taken together with the recent observation of rare loss of function mutations in the enteroviral response gene $I F I H 1$ protective against type 1 diabetes 31 , these findings suggest viral infection (and the nature of the host response to infection) as a putative environmental trigger common to these autoimmune diseases.

\section{3) T and B cell co-stimulation (or co-inhibition)}

This class of molecules controls the strength and nature of the response to $\mathrm{T}$ or $\mathrm{B}$ (immunoglobulin) cell receptor activation by antigens. We observe multiple regions with genes (CTLA4/ICOS/CD28, TNFRSF14, CD80, ICOSLG, TNFRSF9, TNFSF4) from this class of ligand-receptor pairs suggesting fine control of the adaptive immune response might be altered in at-risk individuals.

\section{4) Cytokines, chemokines and their receptors}

Our previous report discussed the function of the 2q11-12 interleukin receptor cluster (IL18RAP, etc), the 3p21 chemokine receptor cluster (CCR5, etc.) and the loci containing IL2/IL21 and IL12A22. We now report additional loci containing TNFSF18 and CCR4.

We estimate that the current celiac disease variants, including the major celiac disease associated $H L A$ variant, $H L A-D Q 2.5 c i s$, less common celiac disease associated haplotypes in the $H L A$ ( $H L A-D Q 8 ; H L A-D Q 2.5$ trans; HLADQ2.2), and the additional 26 definitively implicated loci explain about $20 \%$ of total celiac disease variance, which would represent $40 \%$ of genetic variance, assuming a heritability of 0.5 . A long tail of low effect size common variants, along with highly penetrant rare variants (both at the established loci and elsewhere in the genome), may substantially contribute to the remaining heritability.

We observed different haplotypes within the ETS1 region associated with coeliac disease in Europeans, and SLE in the Chinese population. We further note for some autoimmune diseases studied in European origin populations, that although the same LD block has been associated, the association is with a different haplotype. In some cases, the same variants are associated, but the direction of association is opposite (e.g. rs917997/IL18RAP in celiac disease versus type 1 diabetes). We believe further exploration of these signals may reveal critical differences in the nature of the immune system perturbation between these diseases.

Previous investigators have observed that only a small proportion of GWAS associations are coding variants, and have suggested that these may instead influence regulation of gene expression. Here, we show that over half the celiac disease associated variants are correlated with expression changes in nearby genes. This mechanism is likely to explain the function of some risk variants for other common, complex diseases. Further research, however, is needed to definitively determine at each locus both the celiac disease causal variants and their functional mechanisms.

\section{Supplementary Material}

Refer to Web version on PubMed Central for supplementary material.

\section{Acknowledgments}

We thank Celiac UK for assistance with direct recruitment of celiac disease individuals, and UK clinicians (L.C. Dinesen, G.K.T. Holmes, P.D. Howdle, J.R.F. Walters, D.S. Sanders, J. Swift, R. Crimmins, P. Kumar, D.P. Jewell, S.P.L. Travis, K. Moriarty) who recruited celiac disease blood samples described in our previous studies 1,22 . We thank the genotyping facility of the UMCG (J. Smolonska, P. van der Vlies) for generating part of the GWAS and replication data and the gene expression data; R. Booij and M. Weenstra are thanked for preparation of Italian samples. H. Ahola, A. Heimonen, L. Koskinen, E. Einarsdottir and K. Löytynoja are thanked for their work on 
Finnish sample collection, preparation and data handling, and E. Szathmári, J.B.Kovács, M. Lörincz and A. Nagy for their work with the Hungarian families. Health2000 organization, Finrisk consortium, K. Mustalahti, M. Perola, K. Kristiansson and J. Koskinen are thanked for providing the Finnish control genotypes. We thank D.G. Clayton and N. Walker for providing T1DGC data in the required format. We thank the Irish Transfusion Service and Trinity College Dublin Biobank for control samples; V. Trimble, E. Close, G. Lawlor, A. Ryan, M. Abuzakouk, C. O’Morain, G. Horgan, for celiac sample collection and preparation We acknowledge DNA provided by Mayo Clinic Rochester, and Prof. M. Bonamico and Prof. M. Barbato (Department of Paediatrics, Sapienza University of Rome, Italy) for recruiting individuals. We thank Polish clinicians for recruitment of celiac disease individuals (Z. Domagala, A. Szaflarska-Poplawska, B. Oralewska, W. Cichy, B. Korczowski, K. Fryderek, E. Hapyn, K. Karczewska, A. Zalewska, I. Sakowska-Maliszewska, R. Mozrzymas, A. Zabka, M. Kolasa, B. Iwanczak). We thank M. Szperl for isolating DNA from blood samples provided by Children's Memorial Health Institute (Warsaw, Poland).

Dutch and UK genotyping for the second celiac disease GWAS was funded by the Wellcome Trust (084743 to D.A.vH.). Italian genotyping for the second celiac disease GWAS was funded by the Coeliac Disease Consortium, an Innovative Cluster approved by the Netherlands Genomics Initiative and partially funded by the Dutch Government (BSIK03009 to C.W.) and by the Netherlands Organisation for Scientific Research (NWO, VICI grant 918.66.620 to C.W.). E.G. is funded by the Italian Ministry of Healthy (grant RC2009). L.H.v.d.B. acknowledges funding from the Prinses Beatrix Fonds, the Adessium foundation, and the Amyotrophic Lateral Sclerosis Association. L.F. received a Horizon Breakthrough grant from the Netherlands Genomics Initiative (93519031) and a VENI grant from NWO (ZonMW grant 916.10.135). P.C.D. is a MRC Clinical Training Fellow (G0700545). G.T. received a Ter Meulen Fund grant from the Royal Netherlands Academy of Arts and Sciences (KNAW). The gene expression study was funded in part by COPACETIC (EU grant 201379). This study makes use of data generated by the Wellcome Trust Case-Control Consortium 2 (WTCCC2). A full list of the WTCCC2 investigators who contributed to the generation of the data is available from www.wtccc.org.uk. Funding for the WTCCC2 project was provided by the Wellcome Trust under award 085475 . This research utilizes resources provided by the Type 1 Diabetes Genetics Consortium, a collaborative clinical study sponsored by the National Institute of Diabetes and Digestive and Kidney Diseases (NIDDK), National Institute of Allergy and Infectious Diseases (NIAID), National Human Genome Research Institute (NHGRI), National Institute of Child Health and Human Development (NICHD), and Juvenile Diabetes Research Foundation International (JDRF) and supported by U01 DK062418. We acknowledge the use of BRC Core Facilities provided by the financial support from the Department of Health via the National Institute for Health Research (NIHR) comprehensive Biomedical Research Centre award to Guy's \& St Thomas' NHS Foundation Trust in partnership with King's College London and King's College Hospital NHS Foundation Trust. We acknowledge funding from NIH: DK050678 and DK081645 (to S.L.N.), NS058980 (to R.A.O.); DK57892 and DK071003 (to J.A.M.). Collection of Finnish and Hungarian patients was funded by EU Commission (MEXT-CT-2005-025270), the Academy of Finland, Hungarian Scientific Research Fund (contract OTKA 61868), the University of Helsinki Funds, the Competitive Research Funding of the Tampere University Hospital, the Foundation of Pediatric Research, Sigrid Juselius Foundation, and the Hungarian Academy of Sciences (2006TKI247 to RA). Funding for the Polish samples collection and genotyping was provided by UMC Cooperation Project (6/06/2006/NDON). R.McM is funded by Science Foundation Ireland. C. Núñez has a FIS contract (CP08/0213). The Dublin Centre for Clinical Research contributed to patient sample collection and is funded by the Irish Health Research Board and the Wellcome Trust

Finally we thank all individuals with celiac disease and control individuals for participating in this study.

\section{Appendix METHODS}

Subjects

Written informed consent was obtained from all subjects, with Ethics Committee / Institutional Review Board approval. All individuals are of European ancestry. Affected celiac individuals were diagnosed according to standard clinical, serological and histopathological criteria including small intestinal biopsy. DNA samples were from blood, lymphoblastoid cell lines or saliva. A more detailed description of subjects is provided in Supplementary Information.

\section{GWAS genotyping}

See Table 1. UK(1) case and control genotyping was previously described1,7. Illumina 670Quad and 1.2M-Duo (custom chips designed for the WTCCC2 and comprising Hap550 / 1M and common CNV content) and 610-Quad genotyping was performed in London, Hinxton 
and Groningen. Bead intensity data was normalized for each sample in BeadStudio, R and theta values exported and genotype calling performed using a custom algorithm1,35. A detailed description of genotype calling steps is provided in Supplementary Information.

Quality control steps were performed in the following order: Very low call rate samples and SNPs were first excluded. SNPs were excluded from all sample collections if any collection showed call rates were $<95 \%$ or deviation from Hardy-Weinberg equilibrium $(P<0.0001)$ in controls. Samples were excluded for call rate $<98 \%$, incompatible recorded gender and genotype inferred gender, ethnic outliers (identified by multi-dimensional scaling plots of samples merged with HapMap Phase II data), duplicates and first degree relatives. 22 of 417 SNPs showing apparent association $\left(\mathrm{P}_{\mathrm{GWAS}}<10^{-4}\right.$ ) were excluded after visual inspection of $\mathrm{R}$ theta plots suggested possible bias.

The over-dispersion factor of association test statistics (genomic control inflation factor), $\lambda_{\mathrm{GC}}$, was calculated using observed versus expected values for all SNPs in PLINK.

\section{Follow-up genotyping}

See Table 1. Finnish controls (12) were genotyped on the 610-Quad BeadChip, other samples were genotyped using Illumina GoldenGate BeadXpress assays in London, Hinxton and Groningen. Genotyping calling was performed in BeadStudio for combined cases and controls in each separate collection, with the exception of the Finnish collection, and whole genome amplified samples (89 Irish cases and 106 Spanish controls). Quality control steps were performed as for the GWAS. 131 of 144 SNPs passed quality control and visual inspection of genotype clouds.

\section{SNP association analysis}

Analyses were performed using PLINK v1.0736, mostly using the Cochran-Mantel-Haenzel test. Logistic regression analyses were used to define the independence of association signals within the same linkage disequilibrium block, with group membership included as a factorized covariate

Genotype imputation was performed for samples genotyped on the Hap300 using BEAGLE and CEU, TSI, MEX and GIH reference samples from HapMap3. Association analysis was performed using logistic regression on posterior genotype probabilities, with group membership included as a factorized covariate.

Structured association tests were performed using PLINK, as described using genetically matched cases and controls within collections identified by identity by state similarity across autosomal non-HLA SNPs as described34 (settings --ppc 0.001 --cc, clusters constrained by the 5 collections). Principal components analysis was performed using EIGENSTRAT and a set of 12,810 autosomal non-HLA SNPs chosen for low LD and ancestry information37,38, association tests were corrected for the top 10 principal components and combined using weighted $\mathrm{Z}$ scores.

The fraction of additive variance was calculated using a liability threshold model39 assuming a population prevalence of $1 \%$. Effect sizes and control allele frequencies were estimated from the combined replication panel. Genetic variance was calculated assuming $50 \%$ heritability.

\section{GRAIL analysis}

We performed GRAIL analysis (http://www.broadinstitute.org/mpg/grail/grail.php) using HG18 and Dec2006 PubMed datasets, default settings for SNP rs number submission, and 
the 27 genome-wide significant celiac disease risk loci (most associated SNP) as seeds. As a query we used either associated SNPs, or $101 \times 50$ randomly chosen Hap550 SNP datasets (5050 SNPs, of which 5033 mapped to the GRAIL database).

\section{Identification of Transcriptional Components}

We noted that the power of eQTL studies in humans is limited by substantial observed interindividual variation in expression measurements due to non-genetic factors, and therefore developed a method, Transcriptional Components, to remove a large component of this variation (manuscript in preparation). Expression data from 42,349 heterogeneous human samples hybridized to Affymetrix HG-U133A (GEO accession number: GPL96) or HGU133 Plus 2.0 (GEO accession number: GPL570) Genechips were downloaded 40 (Fig. 1, step 1). Samples missing data for $>150$ probes were excluded, and only probes available on both platforms were analysed, resulting in expression data for 22,106 probes and 41,408 samples. We performed quantile normalization using the median rank distribution 41 and $\log _{2}$ transformed the data - ensuring an identical distribution of expression signals for every sample, discarding previous normalization and transformation steps.

Initial quality control (QC) was performed by applying principal component analysis (PCA) on the sample correlation matrix (pair-wise Pearson correlation coefficients between all samples). The first principal component (PC), explaining 80-90\% of the total variance 42,43, describes probe-specific variance. 6,375 samples with correlation $R<0.75$ of the sample array with this PC were considered outliers of lesser quality and excluded from analysis. We excluded entire GEO datasets where $>25 \%$ of the samples were outliers (probably expression ratios versus a reference, not absolute data). The final dataset comprised 33,109 samples (17,568 GPL96 and 15,541 GPL570 samples), and we repeated the normalization and transformation on the originally deposited expression values of these post-quality control samples.

We next applied PCA on the pairwise 22,106 $\times 22,106$ probe Pearson correlation coefficient matrix assayed on the 33,109 sample dataset (our fast C++ tool, MATool, is available upon request), attempting to simplify the structure of the data. Here, PCA represents a transformation of a set of correlated probes into sets of uncorrelated linear additions of probe expression signals (eigenvectors) that we name Transcriptional Components (TCs). Each TC is a weighted sum of probe expression signals and eigenvector probe coefficients. These TC-scores can be calculated for each observed expression array sample (reflecting the TC activity per sample).

\section{Subjects for expression - genotype correlation}

We obtained peripheral blood DNA and RNA (PAXgene) from Dutch and UK individuals who were disease cases or controls for GWAS studies (Supplementary Table 1). All samples had been genotyped for a common SNP set on Illumina platforms. Analysis was confined to 294,767 SNPs that had a MAF $\geq 5 \%$, call-rate $\geq 95 \%$ and exact HWE $P>0.001$. RNA from the samples was either hybridized to Illumina HumanRef-8 v2 arrays (229 samples, Ref-8v2) or Illumina HumanHT-12 arrays (1,240 samples, HT-12), and raw probe intensity extracted using BeadStudio. The Ref-8v2 samples were jointly quantile normalized and $\log _{2}$ transformed, and similarly for the HT-12 samples. Subsequent analyses were also conducted separately for these datasets, up to the eventual eQTL mapping, that uses a meta-analysis framework, combining eQTL results from both arrays. HT-12 and Ref-8v2 arrays are different, but share many probes with identical probe sequences. Illumina sometimes use different probe identifiers for the same probe sequences - in meta-analysis and Table 3, the label HT-12 was used if both HT-12 and Ref-8v2 had the same sequence. 


\section{Re-mapping of probes}

If probes mapped incorrectly, or cross-hybridized to multiple genomic loci, it might be that an eQTL would be detected that would be deemed a trans-eQTLs. To prevent this, we used a mapping approach versus a known reference that we developed for high-throughput short sequence RNAseq data44. We took the DNA sequence as synthesised for each cDNA probe, and aligned it versus a transcript masked gDNA genome combined with cDNA sequences. A more detailed description of probe re-mapping is provided in Supplementary Information. Probes that did not map, or mapped to multiple different locations were removed.

\section{Affymetrix transcriptional components applied to Illumina expression data}

TC-scores can be inferred in new (non-Affymetrix) datasets for every new individual sample. For the Illumina samples (used for the cis-eQTL mapping), only Illumina probes that could be mapped to any of our 22,106 Affymetrix probes were used (www.switchtoi.com/probemapping.ilmn). The TC-score of sample $i$ for the $f^{\text {th }}$ TC is defined as: TCscore $_{i j}=\sum_{t=1}^{t=n} a_{t i} \times v_{t j}$, where $\mathrm{v}_{\mathrm{tj}}$ is defined as the $t^{\text {th }}$ Affymetrix probe coefficient for the $j^{\text {th }}$ TC; $\mathrm{a}_{t i}$ is the Illumina expression measurement for the $t^{\text {th }}$ mapped probe for sample $i$. We inferred the Illumina TC-scores for the top 1,000 TCs.

\section{Removal of transcriptional component effects from Illumina expression data}

Because our Illumina eQTL dataset $(n=1,469)$ is much less heterogeneous than the Affymetrix dataset $(n=33,109)$, we expect that some TCs will hardly vary. We therefore performed a PCA on the covariance matrix of the top 1,000 inferred TC-scores for the Illumina dataset to effectively compress the TC data into a small set of 'aggregate TCs' (aTCs). As aTCs are orthogonal, we used linear regression to eliminate the effect of the top 50 aTCs. We correlated the TC-scores for each peripheral blood sample with probe expression levels. We then used the resulting residual gene expression data for subsequent cis-eQTL mapping.

\section{cis-eQTL mapping}

We used the residual gene expression data (Fig. 1) in a meta-analysis framework, as described45,46. In brief, analyses were confined to those probe-SNP pairs for which the distance from probe transcript midpoint to SNP genomic location was less than $500 \mathrm{~kb}$. To prevent spurious associations due to outliers, a non-parametric Spearman's rank correlation analysis was performed. When a particular probe-SNP pair was present in both the HT12 and H8v2 datasets, an overall, joint p-value was calculated using a weighted Z-method (square root of the dataset sample number). To correct for multiple testing we controlled the false discovery rate (FDR). The distribution of observed $P$ values was used to calculate the FDR, by permuting expression phenotypes relative to genotypes 1000 times within the HT12 and H8v2 dataset. Finally, we removed any probes from analysis which contain a known SNP (1000Genomes CEU SNP data, April 2009 release).

\section{References}

1. van Heel DA, et al. A genome-wide association study for celiac disease identifies risk variants in the region harboring IL2 and IL21. Nat Genet. 2007; 39:827-9. [PubMed: 17558408]

2. van Heel DA, West J. Recent advances in coeliac disease. Gut. 2006; 55:1037-46. [PubMed: 16766754]

3. Sollid LM, et al. Evidence for a primary association of celiac disease to a particular HLA-DQ alpha/ beta heterodimer. J Exp Med. 1989; 169:345-50. [PubMed: 2909659] 
4. Kim CY, Quarsten H, Bergseng E, Khosla C, Sollid LM. Structural basis for HLA-DQ2-mediated presentation of gluten epitopes in celiac disease. Proc Natl Acad Sci U S A. 2004; 101:4175-9. [PubMed: 15020763]

5. Henderson KN, et al. A Structural and Immunological Basis for the Role of Human Leukocyte Antigen DQ8 in Celiac Disease. Immunity. 2007; 27:23-34. [PubMed: 17629515]

6. Zhernakova A, van Diemen CC, Wijmenga C. Detecting shared pathogenesis from the shared genetics of immune-related diseases. Nat Rev Genet. 2009; 10:43-55. [PubMed: 19092835]

7. Barrett JC, et al. Genome-wide association study and meta-analysis find that over 40 loci affect risk of type 1 diabetes. Nat Genet. 2009

8. Barrett JC, et al. Genome-wide association defines more than 30 distinct susceptibility loci for Crohn's disease. Nat Genet. 2008; 40:955-62. [PubMed: 18587394]

9. Anderson CA, et al. Evaluating the effects of imputation on the power, coverage, and cost efficiency of genome-wide SNP platforms. Am J Hum Genet. 2008; 83:112-9. [PubMed: 18589396]

10. Jacobs KB, et al. A new statistic and its power to infer membership in a genome-wide association study using genotype frequencies. Nat Genet. 2009

11. Wellcome Trust Case Control Consortium. Genome-wide association study of 14,000 cases of seven common diseases and 3,000 shared controls. Nature. 2007; 447:661-78. [PubMed: 17554300]

12. Pe'er I, Yelensky R, Altshuler D, Daly MJ. Estimation of the multiple testing burden for genomewide association studies of nearly all common variants. Genet Epidemiol. 2008; 32:381-5. [PubMed: 18348202]

13. Dudbridge F, Gusnanto A. Estimation of significance thresholds for genomewide association scans. Genet Epidemiol. 2008; 32:227-34. [PubMed: 18300295]

14. Karell K, et al. HLA types in celiac disease patients not carrying the DQA $1 * 05-\mathrm{DQB} 1 * 02$ (DQ2) heterodimer: results from the European Genetics Cluster on Celiac Disease. Hum Immunol. 2003; 64:469-77. [PubMed: 12651074]

15. Raychaudhuri S, et al. Genetic variants at CD28, PRDM1 and CD2/CD58 are associated with rheumatoid arthritis risk. Nat Genet. 2009

16. Raychaudhuri $\mathrm{S}$, et al. Identifying relationships among genomic disease regions: predicting genes at pathogenic SNP associations and rare deletions. PLoS Genet. 2009; 5:e1000534. [PubMed: 19557189]

17. Smyth DJ, et al. Shared and distinct genetic variants in type 1 diabetes and celiac disease. N Engl J Med. 2008; 359:2767-77. [PubMed: 19073967]

18. Coenen MJ, et al. Common and different genetic background for rheumatoid arthritis and coeliac disease. Hum Mol Genet. 2009

19. Hindorff LA, et al. Potential etiologic and functional implications of genome-wide association loci for human diseases and traits. Proc Natl Acad Sci U S A. 2009; 106:9362-7. [PubMed: 19474294]

20. Yu W, Clyne M, Khoury MJ, Gwinn M. Phenopedia and Genopedia: Disease-centered and Genecentered Views of the Evolving Knowledge of Human Genetic Associations. Bioinformatics. 2009

21. Han JW, et al. Genome-wide association study in a Chinese Han population identifies nine new susceptibility loci for systemic lupus erythematosus. Nat Genet. 2009; 41:1234-7. [PubMed: 19838193]

22. Hunt KA, et al. Newly identified genetic risk variants for celiac disease related to the immune response. Nat Genet. 2008; 40:395-402. [PubMed: 18311140]

23. Allen PM. Themis imposes new law and order on positive selection. Nat Immunol. 2009; 10:8056. [PubMed: 19621038]

24. Sato T, et al. Dual functions of Runx proteins for reactivating CD8 and silencing CD4 at the commitment process into CD8 thymocytes. Immunity. 2005; 22:317-28. [PubMed: 15780989]

25. Woolf E, et al. Runx 3 and Runx 1 are required for CD8 T cell development during thymopoiesis. Proc Natl Acad Sci U S A. 2003; 100:7731-6. [PubMed: 12796513]

26. Wang J, Fu YX. LIGHT (a cellular ligand for herpes virus entry mediator and lymphotoxin receptor)-mediated thymocyte deletion is dependent on the interaction between TCR and MHC/ self-peptide. J Immunol. 2003; 170:3986-93. [PubMed: 12682226] 
27. Zamisch M, et al. The transcription factor Ets1 is important for CD4 repression and Runx3 upregulation during CD8 T cell differentiation in the thymus. J Exp Med. 2009

28. Vafiadis P, et al. Insulin expression in human thymus is modulated by INS VNTR alleles at the IDDM2 locus. Nat Genet. 1997; 15:289-92. [PubMed: 9054944]

29. Bonasio R, et al. Clonal deletion of thymocytes by circulating dendritic cells homing to the thymus. Nat Immunol. 2006; 7:1092-100. [PubMed: 16951687]

30. Klein L, Hinterberger M, Wirnsberger G, Kyewski B. Antigen presentation in the thymus for positive selection and central tolerance induction. Nat Rev Immunol. 2009; 9:833-844. [PubMed: 19935803]

31. Nejentsev S, Walker N, Riches D, Egholm M, Todd JA. Rare Variants of IFIH1, a Gene Implicated in Antiviral Responses, Protect Against Type 1 Diabetes. Science. 2009

32. Trynka G, et al. Coeliac disease-associated risk variants in TNFAIP3 and REL implicate altered NF-kappaB signalling. Gut. 2009; 58:1078-83. [PubMed: 19240061]

33. Garner CP, et al. Replication of celiac disease UK genome-wide association study results in a US population. Hum Mol Genet. 2009; 18:4219-25. [PubMed: 19648293]

34. Plenge RM, et al. Two independent alleles at $6 \mathrm{q} 23$ associated with risk of rheumatoid arthritis. Nat Genet. 2007; 39:1477-82. [PubMed: 17982456]

35. Franke L, et al. Detection, imputation, and association analysis of small deletions and null alleles on oligonucleotide arrays. Am J Hum Genet. 2008; 82:1316-33. [PubMed: 18519066]

36. Purcell S, et al. PLINK: A Tool Set for Whole-Genome Association and Population-Based Linkage Analyses. Am J Hum Genet. 2007; 81:559-75. [PubMed: 17701901]

37. Price AL, et al. Principal components analysis corrects for stratification in genome-wide association studies. Nat Genet. 2006; 38:904-9. [PubMed: 16862161]

38. Yu K, et al. Population substructure and control selection in genome-wide association studies. PLoS One. 2008; 3:e2551. [PubMed: 18596976]

39. Risch NJ. Searching for genetic determinants in the new millennium. Nature. 2000; 405:847-56. [PubMed: 10866211]

40. Edgar R, Domrachev M, Lash AE. Gene Expression Omnibus: NCBI gene expression and hybridization array data repository. Nucleic Acids Res. 2002; 30:207-10. [PubMed: 11752295]

41. Bolstad BM, Irizarry RA, Astrand M, Speed TP. A comparison of normalization methods for high density oligonucleotide array data based on variance and bias. Bioinformatics. 2003; 19:185-93. [PubMed: 12538238]

42. Sherlock G. Analysis of large-scale gene expression data. Brief Bioinform. 2001; 2:350-62. [PubMed: 11808747]

43. Alter O, Brown PO, Botstein D. Singular value decomposition for genome-wide expression data processing and modeling. Proc Natl Acad Sci U S A. 2000; 97:10101-6. [PubMed: 10963673]

44. Heap GA, et al. Genome-wide analysis of allelic expression imbalance in human primary cells by high throughput transcriptome resequencing. Hum Mol Genet. 2009

45. Heap GA, et al. Complex nature of SNP genotype effects on gene expression in primary human leucocytes. BMC Med Genomics. 2009; 2:1. [PubMed: 19128478]

46. Franke L, Jansen RC. eQTL analysis in humans. Methods Mol Biol. 2009; 573:311-28. [PubMed: 19763935] 


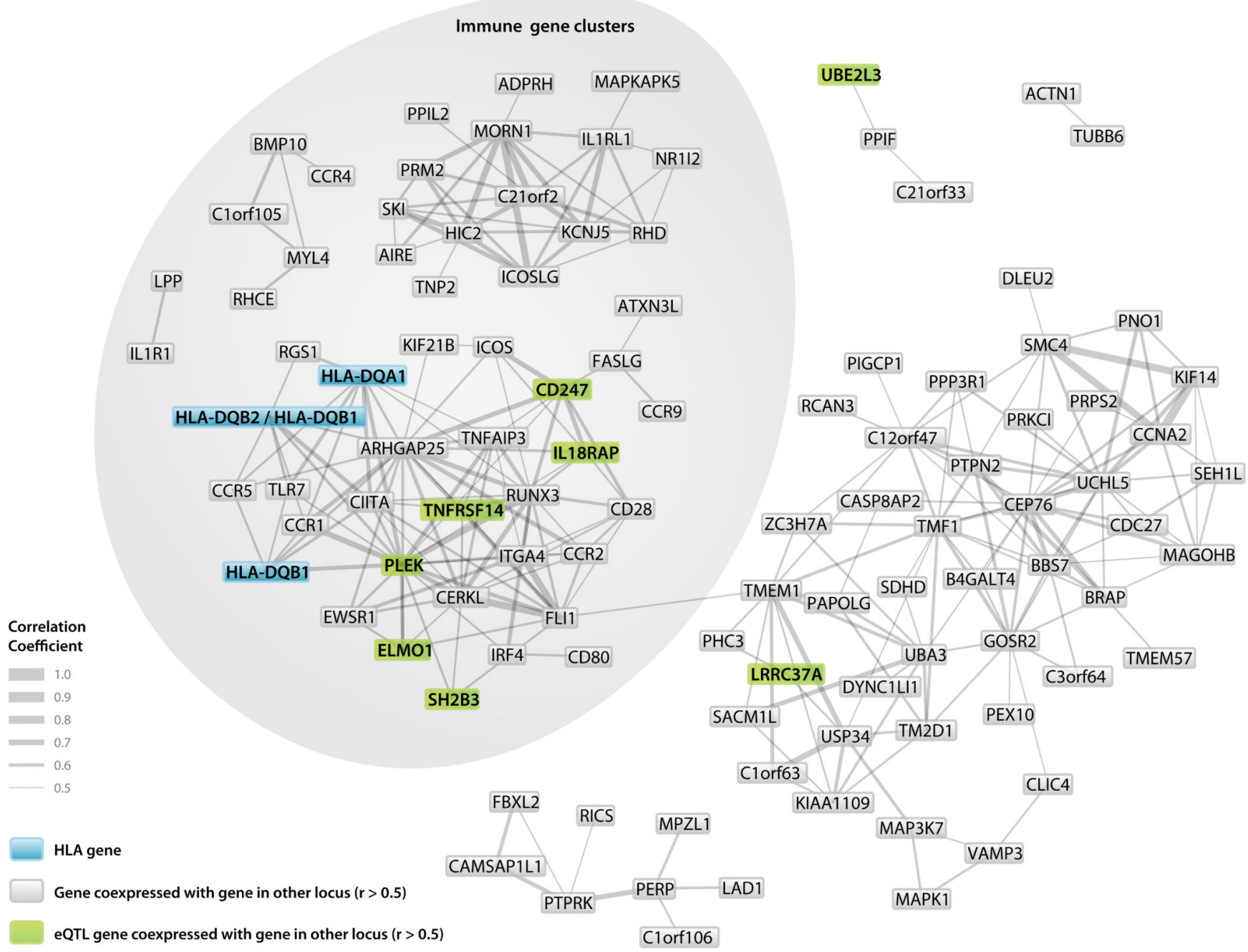

Figure 1. Co-expression analysis of genes mapping to $\mathbf{4 0}$ genome-wide significant and suggestive celiac disease regions in 33,109 heterogenous human samples from the Gene Expression Omnibus

Genes mapping within a $1 \mathrm{Mb}$ window of associated SNPs (Table 2) were tested for interaction with genes from other loci. Interactions with Pearson correlation $>0.5$ shown $\left(\mathrm{P}<10^{-100}\right)$. Only the genes known to contain causal mutations (HLA-DQA1, HLA-DQB1) were analysed from the HLA region, "HLA-DQB2 / HLA-DQB1" is a single expression probeset mapping to both genes. No probe for THEMIS was present on the earlier version of the U133 array, however in a subset analysis of U133 Plus2.0 data, THEMIS is co-expressed in the major immune gene cluster 


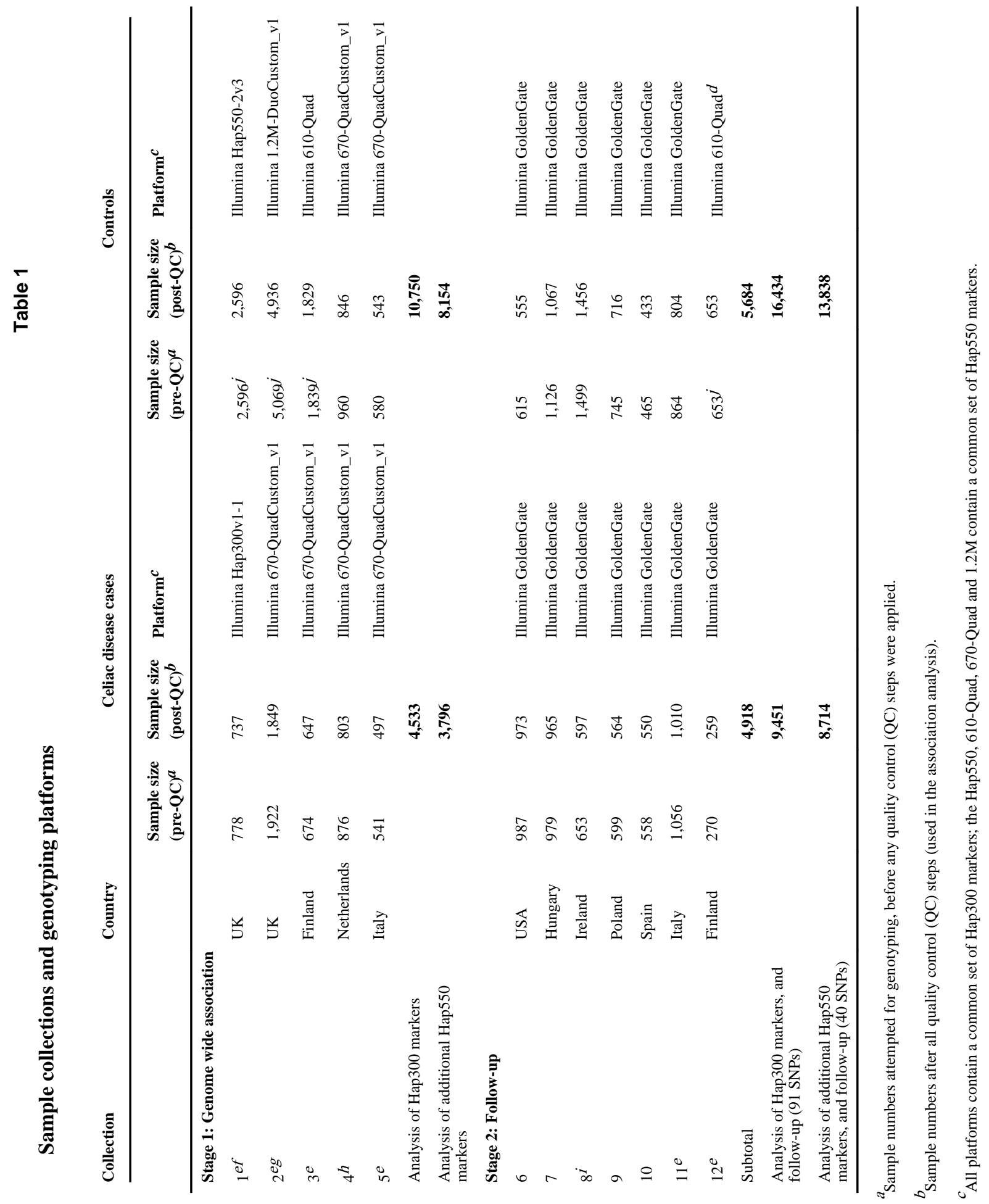




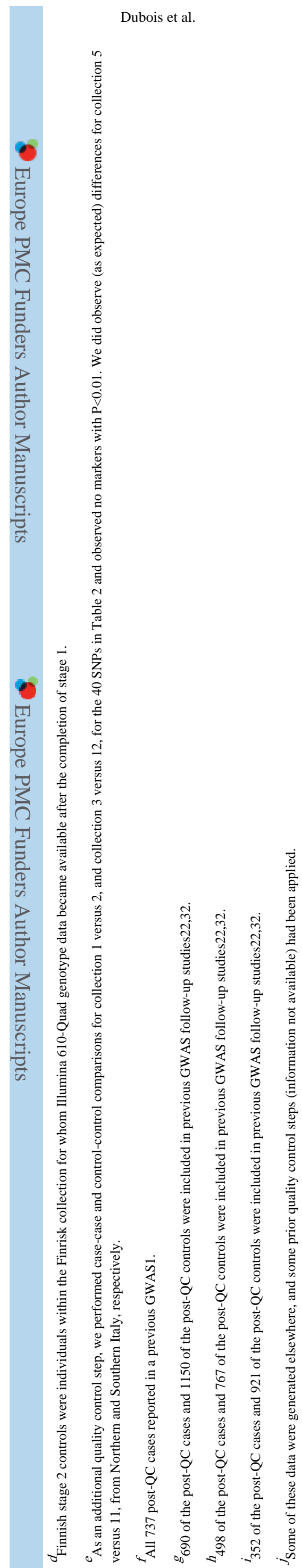




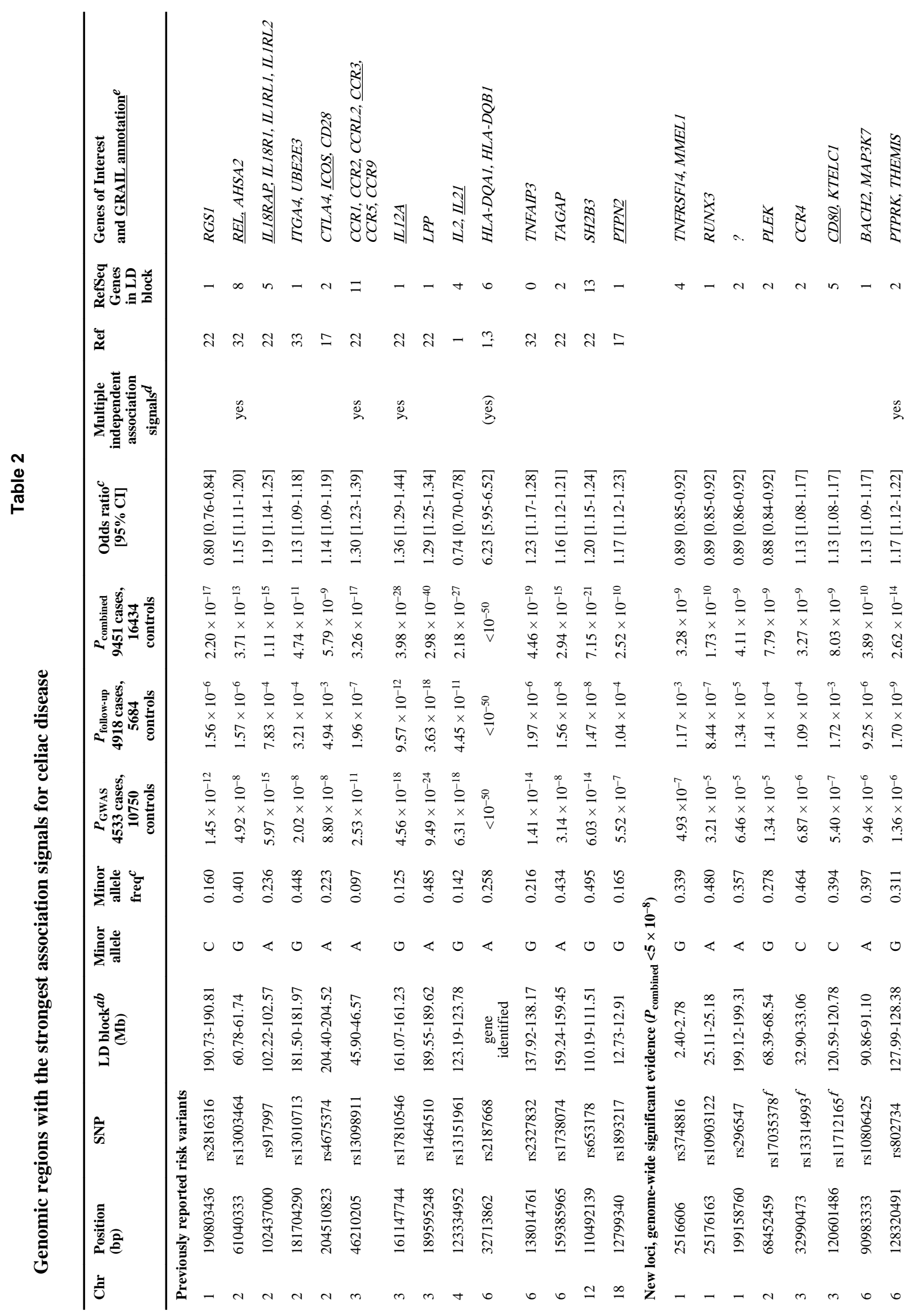


$\frac{m}{10}$

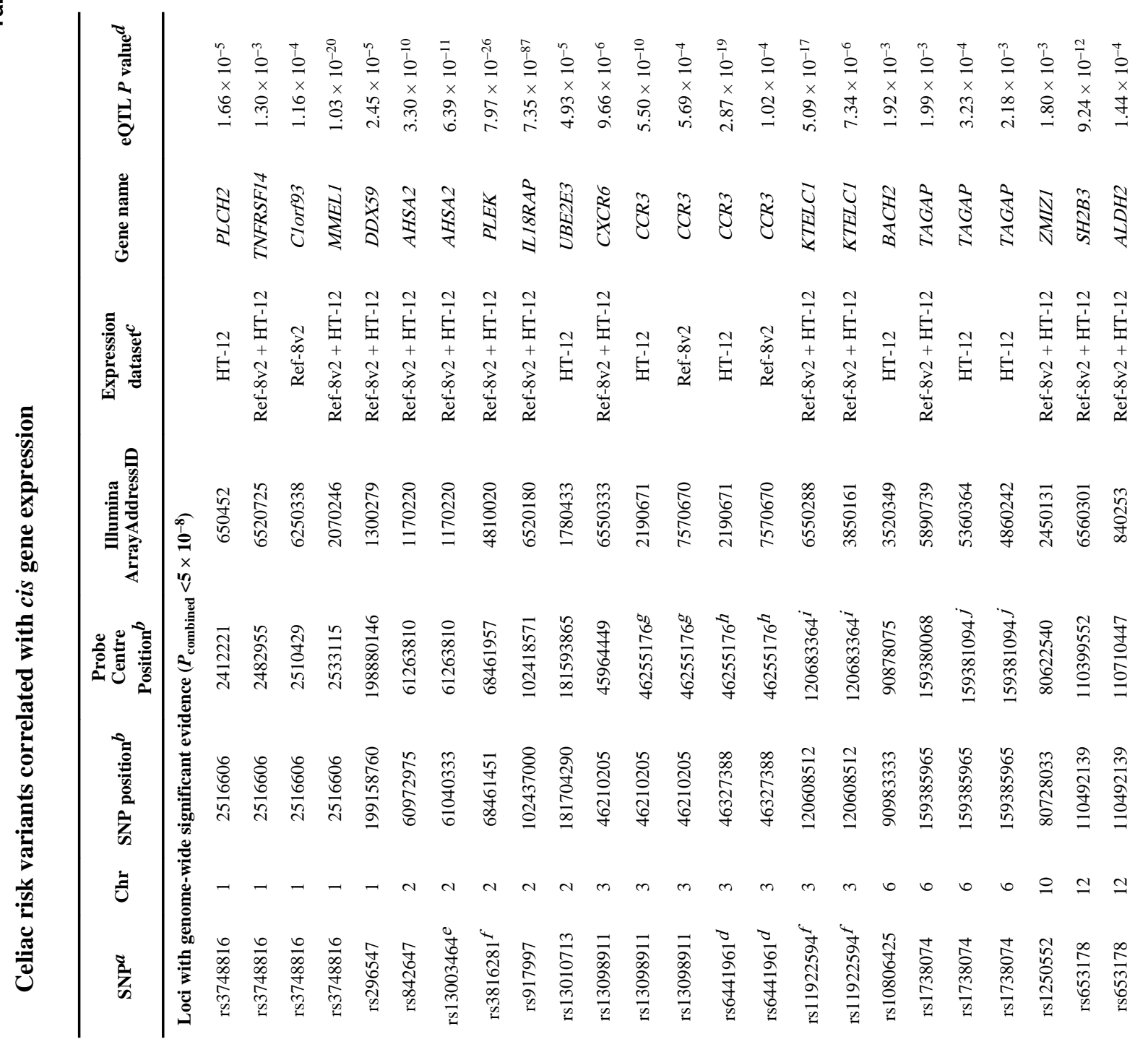




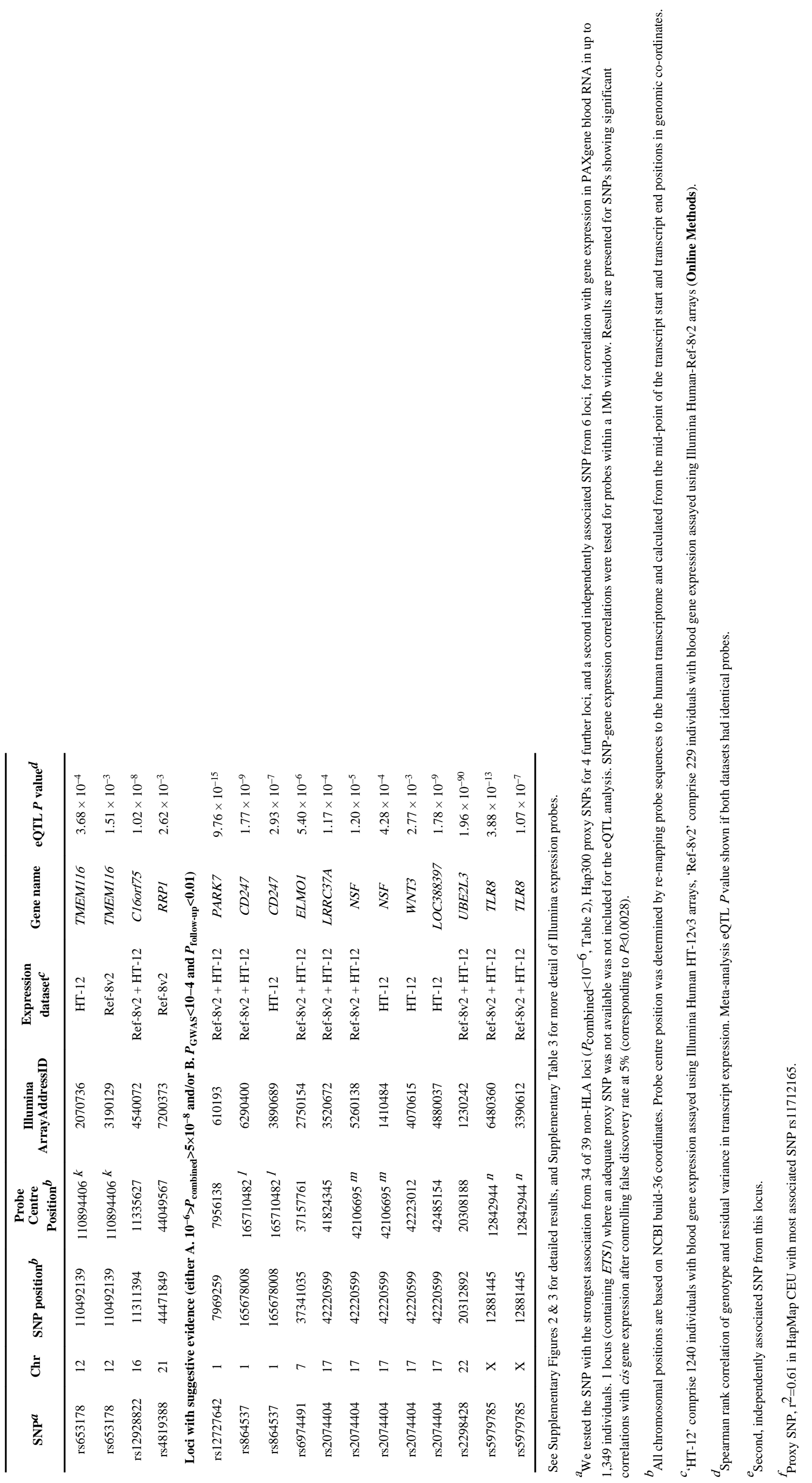




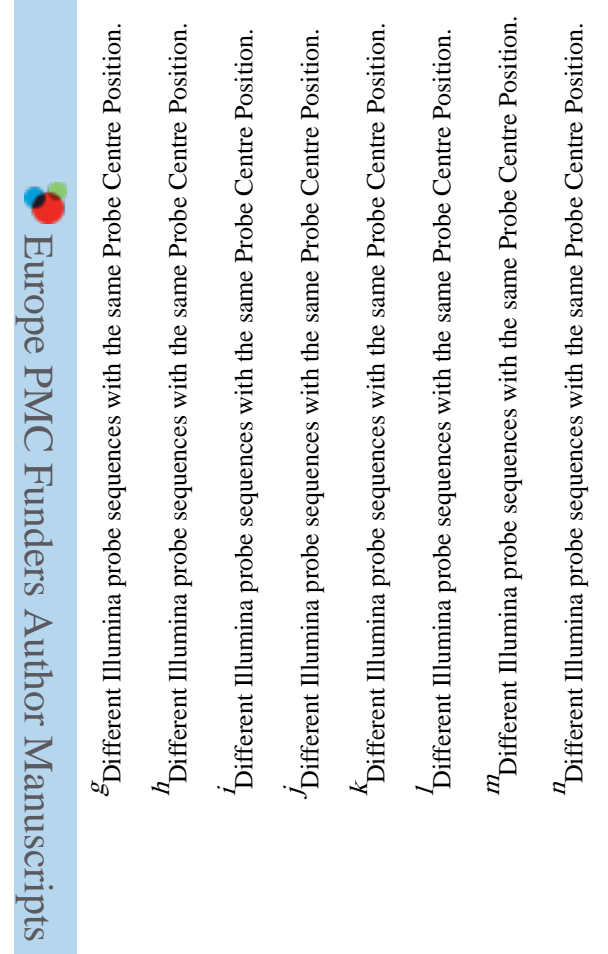

